\title{
Expression Analysis of the Anthocyanin Genes in Pink Spathes of Anthurium with Different Color Intensities
}

\author{
David Gopaulchan, Adrian M. Lennon, and Pathmanathan Umaharan ${ }^{1}$ \\ Department of Life Sciences, Faculty of Science and Technology, The University of the West Indies, \\ St. Augustine Campus, College Road, Republic of Trinidad and Tobago
}

\begin{abstract}
AdDitional INDEX wORDs. flavonoid, phenylpropanoid pathway, gene regulation, western blots, ornamentals, color intensity, Anthurium andraeanum

Abstract. Anthurium (Anthurium andraeanum) is a tropical ornamental valued for its colorful spathe (modified bract) that subtends the inflorescence. The present genetic model for spathe color inheritance in anthurium does not account for differences among the red- and pink-spathed cultivars or for differences in the shades of pink among pink cultivars. To identify the mechanisms responsible for the variation in color and intensity, five genetically defined pinkspathed cultivars, with respect to the $O, R$, and $M$ loci, with varying shade intensities, along with a genetically defined red-spathed cultivar (control), were analyzed at the mRNA, protein, chemical, and phenotypic levels at different spathe development stages. Spathe color values were recorded based on CIE $L^{*} a^{*} b^{*}$ system. Intensity of color ( $L *$, which represents lightness) correlated with the anthocyanin content, with $L$ * showing a strong negative relationship with anthocyanin abundance. Additionally, the red spathe accumulated anthocyanin throughout the spathe developmental stages, whereas the pinks either produced anthocyanin at early stages of development, which decreased as the spathe matured or showed a marked delay in anthocyanin accumulation. The level of anthocyanin closely mirrored flavonoid $3^{\prime}$-hydroxylase $\left(F 3^{\prime} H\right)$ expression but did not correspond with the expression of any of the other genes assayed, chalcone synthase $(C H S)$, flavanone 3-hydroxylase $(F 3 H)$, dihydroflavonol 4-reductase $(D F R)$, and anthocyanidin synthase $(A N S)$. It was found that earlier the expression and higher the rate of expression of $F 3^{\prime} H$ during spathe development, the greater the accumulation of anthocyanin in the spathe. Differences in the $a^{*}$ color space parameter among cultivars also suggests that qualitative differences in color could be mediated through $F 3^{\prime} H$. Other ancillary mechanisms that down regulate $F 3 H, A N S$, and $D F R$ expression levels, evident in some pink cultivars, are discussed.
\end{abstract}

Anthurium has emerged as a popular tropical ornamental in international markets. The cut flower consists of a brilliantly colored modified leaf called the spathe with a protruding, cylindrical inflorescence called the spadix. Spathe color is one of the most attractive features of the cut flower and significantly influences consumer preference of cultivars. Colors range from red, pink, orange, coral, white, green, and brown to spatial bicolors referred to in the industry as obakes. Iwata et al. (1979) demonstrated that anthocyanins were the main pigments responsible for the various spathe colors of anthurium and identified two anthocyanins [cyanidin 3-rutinoside (red) and pelargonidin 3-rutinoside (orange)] in red-, orange-, pink-, and coral-colored spathes. A subsequent study noted that the spathe color and its intensity were determined by both the concentration of cyanidin and ratio of cyanidin to pelargonidin, with red and pink spathes having a ratio over 1.0 and orange and coral spathes having a ratio less than 1.0 (Iwata et al., 1985).

The biosynthetic pathway that leads to anthocyanin formation has been well established and is conserved over a wide range of plants (Fig. 1) (Schijlen et al., 2004). In anthurium,

Received for publication 6 May 2015. Accepted for publication 27 July 2015. We are thankful to Jennifer Avey and her staff at Kairi Blooms Ltd., for donating the cut flowers used in this study. We are also grateful to The University of the West Indies, School for Graduate Studies and Research for granting a Postgraduate Scholarship to D. Gopaulchan. This research was funded by a grant from the Government of Trinidad and Tobago and the University of the West Indies, Campus Research and Publication fund.

${ }^{1}$ Corresponding author. E-mail: pathmanathan.umaharan@sta.uwi.edu.
Collette et al. (2004) cloned and characterized the expression of four genes encoding key enzymes within the anthocyanin biosynthetic pathway, $C H S, F 3 H, D F R$, and $A N S$ and demonstrated that the $C H S, F 3 H$, and $A N S$ genes were coordinately controlled, whereas $D F R$ was controlled by a distinct regulatory mechanism. A subsequent genetic study (Elibox and Umaharan, 2008) showed that three loci, $R, O$, and $M$ determined the major spathe colors in anthurium where the $R$ and $O$ loci controlled whether anthocyanins were produced or not and the $M$ locus determined the type of anthocyanins, whether cyanidin or pelargonidin derivatives. It was postulated that the $R$ and $O$ genes encoded transcription factors that regulated the $C H S, F 3 H$, and $A N S$ genes and the DFR gene, respectively, while $M$ encoded the F3' $\mathrm{H}$ enzyme that converts dihydrokaempferol to dihydroquercetin, a precursor for cyanidin derivatives (Elibox and Umaharan, 2008). More recent studies have shown that $C H S$ is regulated independently of $F 3 H$ and $A N S$ and that the $R$ gene controlled the expression of $F 3 H$ and $A N S$, whereas the $O$ gene controlled the expression of DFR (Avila-Rostant et al., 2011; Gopaulchan et al., 2014). Moreover, a partial sequence of the $F 3^{\prime} H$ gene was cloned and shown to be a likely candidate for $M$ through expression analysis (Gopaulchan et al., 2014).

However, the existing genetic model is inadequate in that it does not account for the range of variation in the shades within a specific spathe color, such as the genetic and molecular bases for differences in shades of reds and pinks. Kamemoto et al. (1988) postulated the dosages of the $O$ and $M$ genes were responsible for the range of shade colors and that pink cultivars were heterozygous for the $M$ and $O$ loci resulting in reduced 


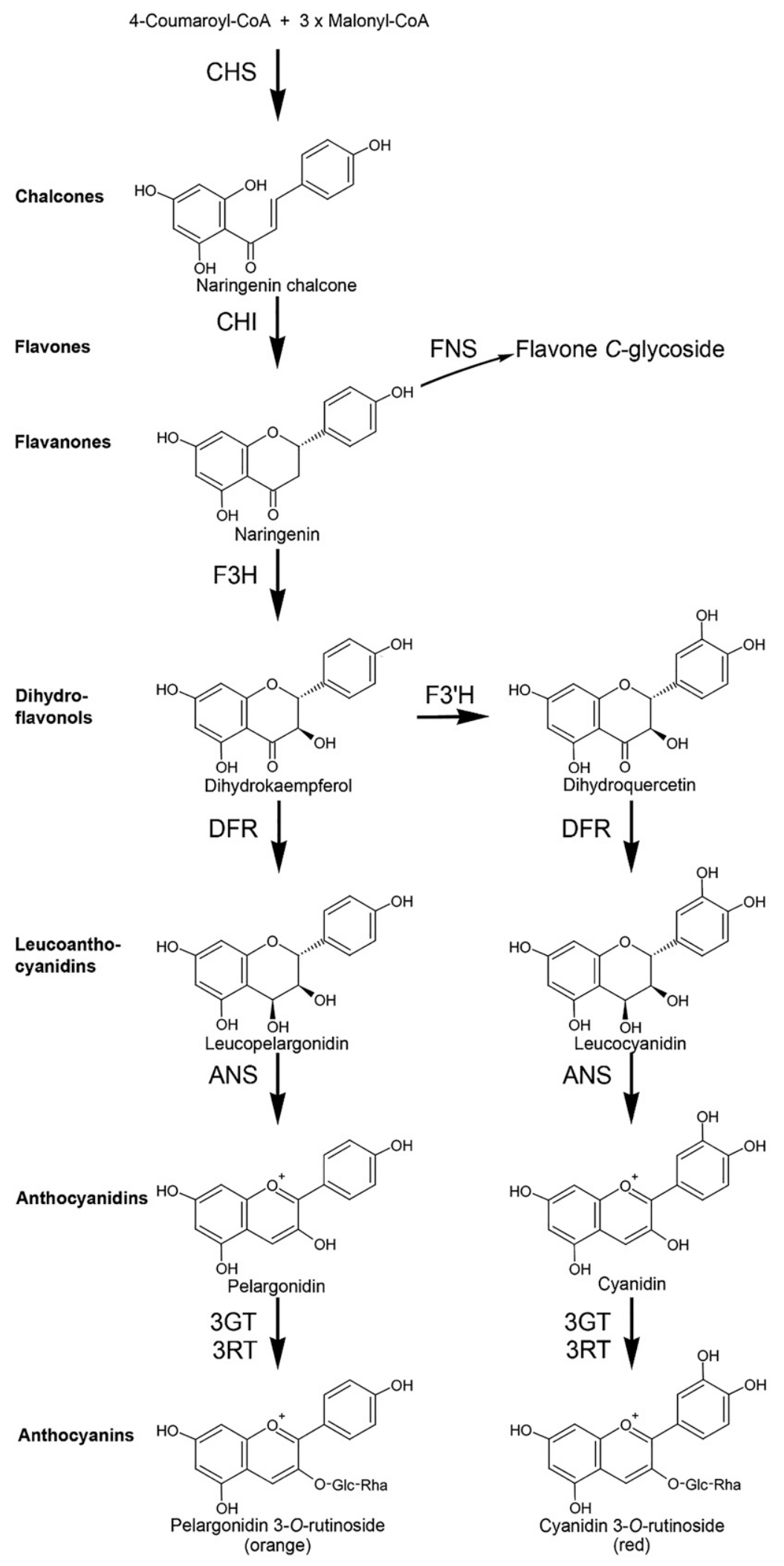

Fig. 1. A simplified diagram of the anthocyanin biosynthetic pathway in Anthurium andraeanum; chalcone synthase $(\mathrm{CHS})$, chalcone isomerase $(\mathrm{CHI})$, flavone synthase $(F N S)$, flavanone 3hydroxylase, flavonoid $3^{\prime}$-hydroxylase $\left(F 3^{\prime} H\right)$, dihydroflavonol 4-reductase $(D F R)$, anthocyanidin synthase $(A N S)$, UDP-glucose:flavonoid 3-O-glucosyltransferase (3GT), and 3-rhamnosyl transferase $(3 R T)$. anthocyanin accumulation. However, Elibox and Umaharan (2008) observed cultivars with pink spathes were generally $O \_R r$ and suggested the $R$ gene exerted a strong dosage effect, depending on the number of dominant alleles it possessed; this was supported by the findings of Avila-Rostant et al. (2011). Collette et al. (2004) found the transcript abundance of the anthocyanin biosynthetic genes peaked late during spathe development in pink spathes compared with red spathes and suggested that temporal expression differences accounted for the differences in the shades of color. Recent studies clearly show that $F 3^{\prime} H$ expression mirrored the accumulation of anthocyanins in red spathes and suggested that the regulation of the $M$ gene may serve as a key point in the control of anthocyanin biosynthesis (Gopaulchan et al., 2014).

The aim of this research was to investigate the underlying molecular mechanism responsible for shade color variation represented by red and pink spathe colored cultivars. To accomplish this, the transcript levels of the $C H S, F 3 H, A N S, D F R$, and $F 3^{\prime} H$ genes were examined at different stages of spathe development using reverse transcription quantitative real-time PCR (RT-qPCR) in five pink-spathed cultivars, with different color shade intensities and representing known genotypes at the $O, R$, and $M$ loci. The expression levels of CHS, F3H, DFR, and ANS proteins were also studied using western hybridization and the results were correlated with anthocyanin content and phenotype.

\section{Materials and Methods}

Plant material. Five pink-spathed cultivars of anthurium, with varying shade color intensity ranging from pale pink to dark pink, were used in this experiment, along with a red-spathed cultivar as a positive control (Table 1). Anthurium cut flowers (the spadix plus the subtending spathe) were collected from mature plants (3 to 4 years old) grown in shade houses with $75 \%$ shade and $\approx 12$-h daylength at Kairi Blooms Ltd., a commercial anthurium farm located at Carapo village, Trinidad. Samples were harvested between 0600 to $0800 \mathrm{HR}$ from spathe development stages 2 (cut flower first visible), 3 (cut flower protrudes from the leaf sheath), 4-1 (peduncle extends to 3-4 cm in length), 4-5 (peduncle fully extended and true flowers evident on the base of the spadix), and 6 (spathe newly opened and fully expanded) (Collette et al., 2004) and immediately stored in liquid nitrogen.

Color determination. Spathe color values were determined using a Chroma Meter 
Table 1 . The putative genotypes with respect to the $O, R$, and $M$ loci for Anthurium andraeanum cultivars (Elibox and Umaharan, 2008).

\begin{tabular}{llcc}
\hline Cultivar & Phenotype & Genotype for $O$ and R & Genotype for $M$ \\
\hline Success & Red & OoRR & $M m$ \\
Lydia & Pink & OORr & $M M$ \\
Sonata & Pink & OORr & $M M$ \\
Spirit & Pink & OoRR & $M m$ \\
Sweety & Dark pink & OoRR & $M m$ \\
Cheers & Pale pink & - & - \\
\hline
\end{tabular}

CR-400 (Konica Minolta Sensing Americas, Ramsey, NJ) based on the CIE $L^{*} a^{*} b^{*}$ system where $L^{*}$ referred to lightness, and $a^{*}$ and $b^{*}$ represented the color component dimensions. The meter was calibrated with a white calibration plate (Konica Minolta Sensing Americas). Results were based on three biological replicates (three distinct spathes) with five random measurements per replicate.

EXTRACTION AND QUANTIFICATION OF TOTAL ANTHOCYANIN. Spathe extract was prepared by incubating the spathe tissue $(0.2 \mathrm{~g})$ in $1 \mathrm{~mL}$ of methanol: acetic acid $(70: 3, \mathrm{v} / \mathrm{v})$ overnight at room temperature. The sample was centrifuged at $16,000 g_{\mathrm{n}}$ for $1 \mathrm{~min}$ and the supernatant was stored. The pellet was subjected to a second extraction with $1 \mathrm{~mL}$ of methanol: acetic acid $(90: 1, \mathrm{v} / \mathrm{v})$ as described before. The supernatants were pooled, evaporated under vacuum to dryness, rehydrated in methanol: acetic acid $(80: 2, \mathrm{v} / \mathrm{v})$, and stored at $-80{ }^{\circ} \mathrm{C}$ until analysis. The total anthocyanin content was determined using the $\mathrm{pH}$ differential method as described by Lee et al. (2005). Absorbencies were measured with an ultraviolet-visible (UV-Vis) spectrophotometer (GENESYS 10; Thermo Fisher Scientific, Waltham, MA) and expressed as cyanidin-3-glucoside equivalent. Results were represented as the mean of three biological replicates.

RNA ISOLATION AND REVERSE TRANSCRIPTION QUANTITATIVE REAL-TIME PCR. Total RNA was extracted from the spathe tissue using the RNAqueous Kit with the Plant RNA Isolation Aid (Applied Biosystems, Foster City, CA) according to the manufacturer's instructions. The RNA was treated with Turbo DNA-free (Applied Biosystems) and quantified using a UV-Vis spectrophotometer (NanoDrop 2000c; Thermo Fisher Scientific). Denatured agarose gel electrophoresis was used to verify the integrity of the purified RNA. The RNA $(1 \mu \mathrm{g})$ was used to synthesize cDNA using the High Capacity cDNA Reverse Transcription Kit (Applied Biosystems) as described by the manufacturer. The cDNA samples were diluted 1 in 5 with nuclease-free water for RT-qPCR reactions.

RT-qPCR assays were performed with Power SYBR Green PCR Master Mix (Applied Biosystems) in 96-well plates and ran on with a 7500 Real-Time PCR System (Applied Biosystems). Primers were designed for each target with Primer Express 3.0.1 (Applied Biosystems) and amplification assays were optimized (Table 2). To control and assess for genomic DNA contamination, primers were designed to target different exons (when possible), and minus reverse transcription control (RT-minus) reactions were made. Reactions were prepared in $10 \mu \mathrm{L}$ volumes containing $1 \mu \mathrm{L}$ of template, $0.5 \mu \mathrm{L}$ of each amplification primer, $5 \mu \mathrm{L}$ of master mix, and $3 \mu \mathrm{L}$ of Milli-Q water. Each PCR reaction was performed in triplicate for three biological replicates, with the inclusion of a pooled RT-minus reaction and a no-template control. The reactions were initially heated to $95{ }^{\circ} \mathrm{C}$ for $10 \mathrm{~min}$, followed by 40 cycles of $95^{\circ} \mathrm{C}$ for
$15 \mathrm{~s}$, and $63{ }^{\circ} \mathrm{C}$ for $1 \mathrm{~min}$. A melting curve analysis ranging from 60 to $95^{\circ} \mathrm{C}$ was performed to verify the specificity of the amplicons and the quantitative cycle $(\mathrm{Cq})$ values were automatically determined using the 7500 System Sequence Detection Software 2.0.6 (Applied Biosystems). Cq values were converted to relative quantities using the respective amplification efficiency (Rieu and Powers, 2009) and normalized to the expression of cyclophilin $(C Y P)$ and ubiquitin family protein (UBQ5) (Table 2) as described by Gopaulchan et al. (2013).

ISOLATION OF TOTAL PROTEIN AND IMMUNOLOGICAL ASSAYS. TO a prechilled mortar and pestle, 0.2 to $0.3 \mathrm{~g}$ of spathe tissue was added and ground to a powder in liquid nitrogen. Protein extraction buffer [ $1 \mathrm{~mL}$ ( $50 \mathrm{~mm}$ MOPS, $250 \mathrm{~mm}$ sucrose, $10 \mathrm{~mm}$ $\mathrm{NaCl}, 5$ mм EDTA pH 8.0, $1 \mathrm{~mm} \mathrm{MgCl}_{2}, 1.5 \%(\mathrm{w} / \mathrm{v})$ polyvinylpyrrolidone, $0.5 \%(\mathrm{v} / \mathrm{v}) 2 \beta$ mercaptoethanol, $\mathrm{pH}$ 7.4)] was added and the sample was ground until it was completely homogenized. The homogenate was transferred to a $1.5-\mathrm{mL}$ microfuge tube and centrifuged at $15,000 \mathrm{~g}_{\mathrm{n}}$ for $1 \mathrm{~min}$ and the supernatant was stored at $-80{ }^{\circ} \mathrm{C}$. The proteins were quantified using the Bradford (1976) protein quantification assay with bovine serum albumin (Sigma-Aldrich, St. Louis, $\mathrm{MO})$ as the standard protein.

The proteins $(10 \mu \mathrm{g})$ were separated on a $12 \%$ SDS polyacrylamide gel (Laemmli, 1970) using an electrophoresis system (Mini-Protean 3; Bio-Rad Laboratories, Hercules, CA) and transferred to $0.45-\mu \mathrm{m}$ nitrocellulose membranes (Sigma-Aldrich) (Harlow and Lane, 1988) with a Mini TransBlot Electrophoretic Transfer Cell (Bio-Rad Laboratories). Electroblotted membranes were stained with Ponceau $\mathrm{S}$ $[0.2 \%(\mathrm{w} / \mathrm{v})$ Ponceau S, $10 \%(\mathrm{w} / \mathrm{v})$ citric acid] to evaluate protein transfer efficiency. Membranes were blocked with 5\% $(\mathrm{w} / \mathrm{v})$ fat-free milk powder in PBS-Tween $[150 \mathrm{~mm} \mathrm{NaCl}$, $7.5 \mathrm{~mm} \mathrm{Na}_{2} \mathrm{HPO}_{4}, 2.5 \mathrm{~mm} \mathrm{NaH} \mathrm{PO}_{4}, 0.1 \%$ Tween $20(\mathrm{v} / \mathrm{v})$ (Sigma-Aldrich), $\mathrm{pH}$ 7.2] for $2 \mathrm{~h}$ on a shaking platform at room temperature and incubated with anti-CHS, - F3H or -ANS sera (1:10,000 final concentration), or purified anti-DFR serum [1:500 (equivalent to $1: 6250$ crude serum)] as described by Gopaulchan et al. (2014) in PBS-Tween overnight on a shaking platform at $4{ }^{\circ} \mathrm{C}$. Membranes were subsequently incubated with ImmunoPure Antibody Donkey Anti-Rabbit IgG (Pierce Protein Research Products, Rockford, IL) at 1:20,000 (final concentration) in PBS-Tween for $1 \mathrm{~h}$ at room temperature.

Visualization of bound antibodies were performed with chemiluminescent substrate (SuperSignal West Pico, Pierce Protein Research Products) according to the manufacturer's instructions and recorded on CL-XPosure Film (Pierce Protein Research Products). Images of blots were acquired with the UVP Gel/Doc-It 300 imaging system with UV to White Light Converter (UVP, Upland, CA) and protein band intensities were estimated using ImageJ 1.46r (Schneider et al., 2012). Due to the rapid proteolytic degradation of $\mathrm{F} 3 \mathrm{H}$ protein into two distinct bands, the quantity of $\mathrm{F} 3 \mathrm{H}$ was determined as the sum of the two band intensities (Gopaulchan et al., 2014; Lukačin et al., 2000; Pelletier et al., 1999).

Statistical analysis. Data on color $\left(L^{*} a^{*} b^{*}\right)$, total anthocyanin content, and the relative quantities of gene expression were analyzed using analysis of variance using the statistical software NCSS (version 2009; NCSS, Kaysville, UT) and means were separated with Fisher's least significant difference test. Relationships between datasets were investigated using Pearson's correlation coefficients. 


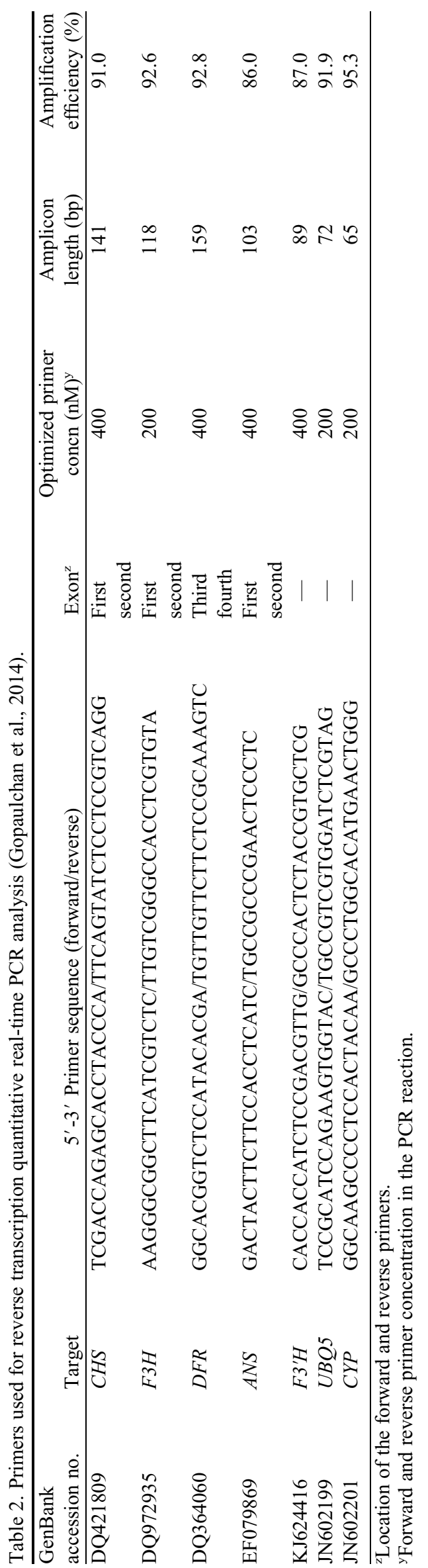

\section{Results}

Chromospectral analysis. Significant differences $(P<$ $0.05)$ in the $L^{*} a^{*} b^{*}$ color space dimensions were recorded among cultivars and developmental stages (Fig. 2). Of these, $L^{*}$ closely reflected the visual intensities of color at stage 6 with the red cultivar, Success, recording the lowest $L^{*}$, followed by 'Sweety' (intermediate value); 'Sonata', 'Lydia', and 'Spirit' (high); and 'Cheers' (very high) in that order. Hence, the darker the shade of color the lower the $L^{*}$ value. Three trends were observed for $L^{*}$ over the development stages for the various anthurium cultivars: 1) $L^{*}$ values decreased from stages 2 to 6 , albeit at different rates, in 'Success', 'Sweety', 'Spirit', and 'Cheers'; however, the decrease was not significant in 'Cheers'. The steeper the rate of decline, the darker the shade of pink obtained at full maturity (stage 6) of the spathe. 2) In 'Sonata', $L^{*}$ decreased significantly $(P<0.05)$ between stages 2 and 4-1, but remained constant thereafter. 3) In 'Lydia', $L^{*}$ decreased significantly $(P<0.05)$ from stages 2 to $4-1$, but showed an increase at stage 6 . These differences influenced the final shade intensity of the cultivars.

The $a^{*}$ color component, which measured the color of the spathes between red/magenta and green (negative $a^{*}$ values indicated green and positive $a^{*}$ values indicated red), was significantly $(P<0.05)$ higher at full maturity (stage 6$)$ in 'Success' and 'Sweety', intermediate in 'Lydia', 'Sonata', and 'Spirit', and low in 'Cheers'; an order that was reminiscent of $L^{*}$. However, there was no correlation $(P>0.05)$ between $L^{*}$ and $a^{*}$ values, suggesting that there was not only quantitative differences in the spathe colors among the pink cultivars, but also qualitative differences. Although the $b^{*}$ color space parameter was significantly $(P<0.05)$ different among cultivars and stages, no specific trend was observed between the red and pink spathes nor between the intensity of shade among the pinks (data not shown).

Total ANTHOCYANIN ACCUMULATION/ABUndanCE. The effects of cultivar, stage and interaction between cultivar, and stage were significant $(P<0.05)$ for the total anthocyanin levels (Fig. 3). The anthocyanin content in the red cultivar, Success, was greater than all the pink cultivars in the mature spathe (stage 6). Among the pink cultivars, Sweety (dark pink) had more $(P<0.05)$ anthocyanins than the other pink cultivars at stage 6 . On average there was an increase in anthocyanin levels from stages 2 to 6 among the pink-spathed cultivars, although there were cultivar differences in the rate of increase (Fig. 3).

'Success' recorded anthocyanins as early as stage 2, which increased at a rapid rate up to stage 6 , thus resulting in the dark red pigmentation. There was evidence of temporal differences in the accumulation of anthocyanins among the pinks. Anthocyanins were evident as early as stage 2 in 'Sweety', which continued to increase up to stage 6, albeit at a slower rate than 'Success' resulting in a dark pink. Although anthocyanin accumulation started as early as stage 2 and stage 3 in 'Lydia' and 'Sonata' respectively, they accumulated anthocyanin at a slower rate up to stage 4-5 and decreased thereafter, while in 'Spirit', anthocyanin accumulation was further delayed up to stage 4-1, but continued to increase at an intermediate rate up to stage 6. 'Lydia', 'Sonata', and 'Spirit' can be regarded as intermediate pinks. 'Cheers', which started accumulation of anthocyanin the latest (at stage 4-5), also increased slowly up to stage 6 and had the lightest pink. The anthocyanin content significantly correlated with the $L^{*}(r=-0.68, P<0.05)$ and $a^{*}$ 
A
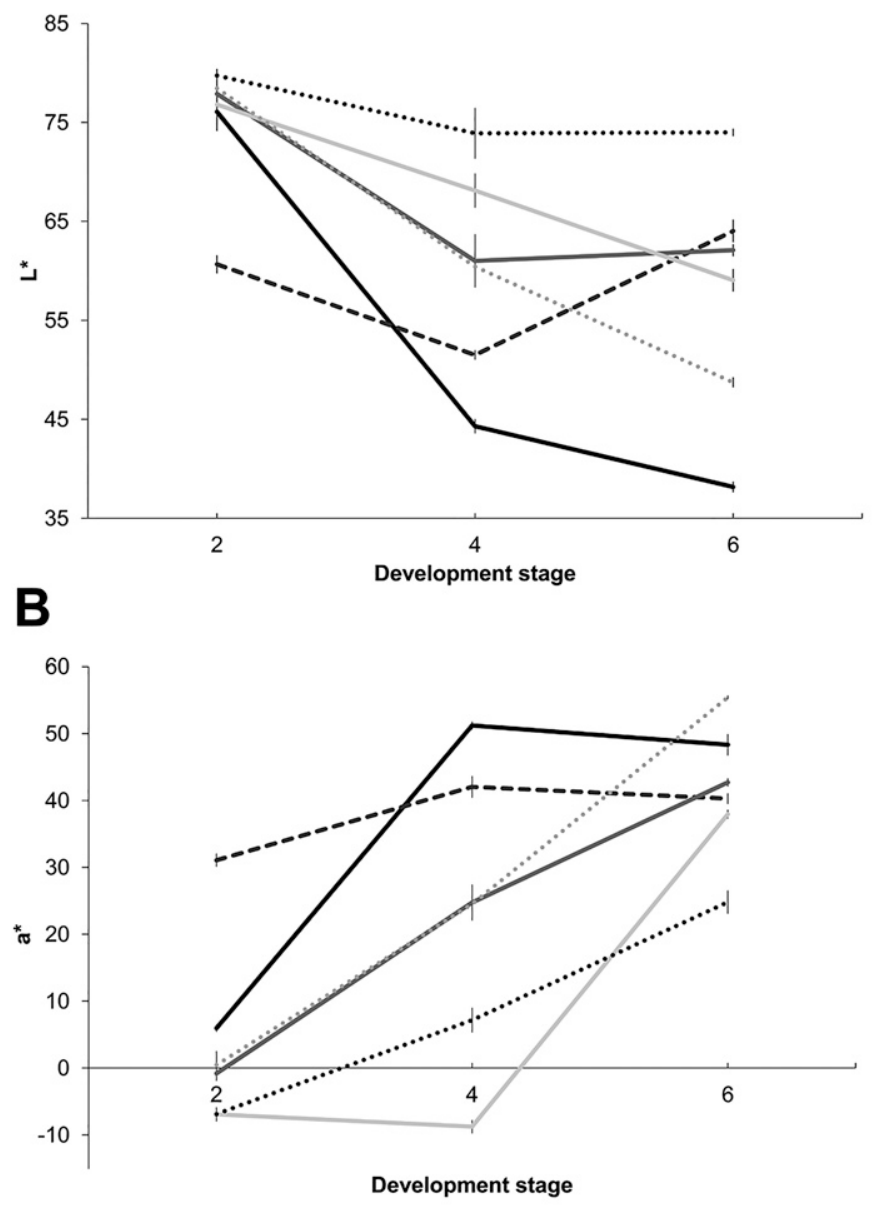

$\begin{array}{lll}\text {-Success } & \text {--Lydia } & \text {-Sonata } \\ \text {-Spirit } & \text {.... Sweety } & \text {..... Cheers }\end{array}$

Fig. 2. Spathe colors for six Anthurium andraeanum cultivars, Success, Lydia, Sonata, Spirit, Sweety, and Cheers were determined at three development stages $(2,4-1$, and 6$)$ using the CIE $L^{*} a^{*} b^{*}$ color system. (A) The $L^{*}$ color space dimension represents the lightness of the spathe where lower $L^{*}$ values indicate greater color intensity and darker shade, and (B) the $a^{*}$ color value measures the position between red/magenta and green where positive values indicate magenta and negative values indicate green. Results are based on three biological replicates with five random measurements per replicate and error bars show the SE.

$(r=0.67, P<0.05)$ (Fig. 4A and B) color space dimensions, indicating its role in shade intensity and quality of color in the spathes.

RT-QPCR ANALYSIS. The effect of cultivar, development stage, and interaction between cultivar $\times$ development stages were significant $(P<0.05)$ for all the genes (Fig. 5). The DFR and $F 3^{\prime} H$ genes showed low levels of overall expression compared with the other genes. Among the pink cultivars at stage 2, the average transcript abundance for $C H S, F 3 H$, and $A N S$ were $3.9,1.5$, and 3.6 times greater than $D F R$, respectively, and $178,66.5$, and 161.6 times greater than $F 3^{\prime} H$, respectively. The effect of developmental stage was most noticeable for DFR, CHS, and ANS transcript abundance in the pink cultivars, with transcript levels declining to $13 \%, 17 \%$, and $50 \%$ at full maturity (stage 6) respectively, as compared



Fig. 3. Total anthocyanin content in the spathe tissue of Anthurium andraeanum cultivars Success, Lydia, Sonata, Spirit, Sweety, and Cheers at spathe development stages 2, 3, 4-1, 4-5, and 6. Anthocyanin levels are expressed as cyanidin-3-glucoside equivalent. Values indicate the mean of three biological replicates and the error bars show the SE.

with stage 2. The rate of decline in transcript abundance from stage 2 to 4-1 to 6 was remarkably linear for these genes $\left(\mathrm{CHS}_{-}\right.$ $\mathrm{b}=-2.0, R^{2}=0.98 ; D F R-\mathrm{b}=-0.51, R^{2}=0.99 ; A N S-\mathrm{b}=-1.01$, $\left.R^{2}=0.95\right)$, with some minor deviations depending on the cultivar.

Although cultivar differences for transcript abundance at full maturity was significant for $C H S, F 3 H, A N S$, and $D F R$, the differences did not correspond with the color intensity of the spathes $\left(L^{*}\right)$ nor the anthocyanin content. In contrast, in the case of $F 3^{\prime} H$ (Fig. 4C and D), there was a strong negative correlation $(r=-0.79, P<0.05)$ between transcript abundance and $L^{*}$, and a strong positive correlation between transcript abundance and total anthocyanin content $(r=0.70, P<0.05)$, indicating a strong correspondence between $F 3^{\prime} H$ expression, anthocyanin accumulation, and darkening of shade of pink. Although there were significant differences $(P<0.05)$ in the mRNA levels between cultivars for all genes, the effect was particularly pronounced for $F 3^{\prime} H$ (Fig. 5). Notably, the $F 3^{\prime} H$ expression was drastically lower in the pink cultivars compared with the red cultivar, Success. The transcript abundance for $F 3^{\prime} H$ in 'Sweety' was $14 \%$ of 'Success', while for the other pinks on average was $4 \%$ of 'Success'. In 'Cheers', the lightest of the pinks, $F 3^{\prime} H$ expression was greatly reduced in comparison with the other pink cultivars. Furthermore, $F 3^{\prime} H$ transcript levels over developmental stages showed a trend similar to $L^{*}$ and total anthocyanin. It increased through the developmental stages but more steeply between stages 4-1 and 6 in 'Success', 'Sweety', and 'Spirit', in that order, but showed a declining trend in the lighter pink cultivars (Sonata, Lydia, and Cheers), accounting for the cultivar $\times$ developmental stage interaction.

IMMUNOLOGICAL ANALYSIS. To investigate the patterns of anthocyanin enzyme accumulation, anti-CHS, -F3H, -DFR, and -ANS antibodies were used to perform immunoblot analysis on crude protein extracts from the spathe tissue at development stages 3, 4-1, 4-5, and 6 (Fig. 6). In 'Success', the CHS, F3H, DFR, and ANS proteins were readily detectable in all spathe development stages though reduced levels were generally recorded at stage 6. The trend was similar in 'Sweety', except that the protein levels of CHS, F3H, DFR, and ANS generally were slightly less compared with 'Success' particularly in the early developmental stages of the spathe. In 'Lydia' and 'Sonata', the CHS, DFR, and ANS proteins were easily detected between 

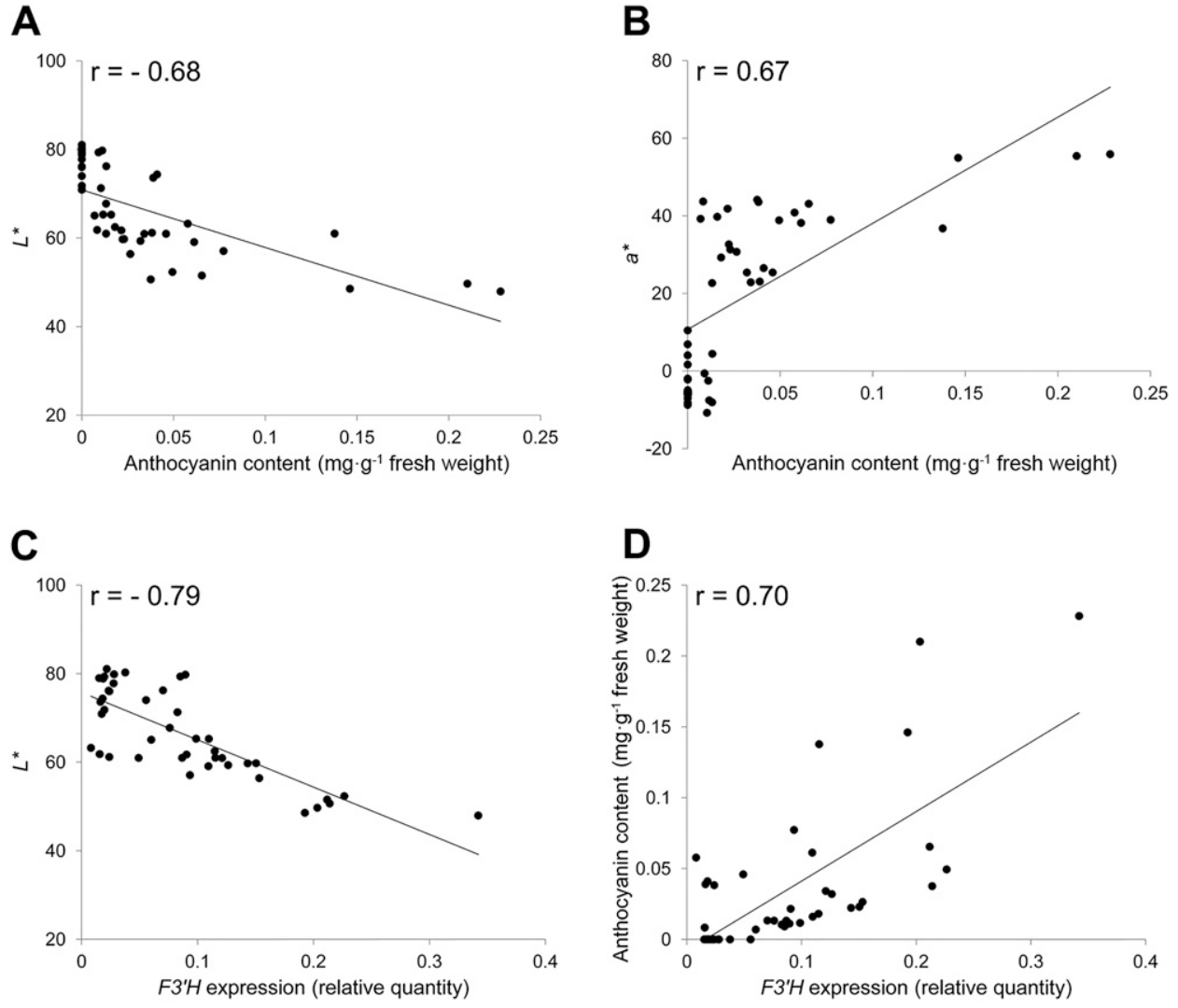

Fig. 4. Correlations among data sets of Anthurium andraeanum cultivars Lydia, Sonata, Spirit, Sweety, and Cheers at spathe development stages 2, 4-1, and 6 were determined at $P<0.05$ significance. Correlation coefficients (r) are shown for all data. (A) The intensity of spathe color $\left(L^{*}\right)$ showed a strong negative relationship with anthocyanin abundance, $(\mathbf{B}) a^{*}$ color space dimension significantly correlated $(P<0.05)$ the anthocyanin levels, (C) the expression of flavonoid 3'-hydroxylase $\left(F 3^{\prime} H\right)$ showed a strong negative relationship with spathe lightness $\left(L^{*}\right)$, and (D) $F 3^{\prime} H$ expression displayed a strong positive correlation between transcript abundance and total anthocyanin content in pink cultivars at development stages 2, 4-1, and 6.

stages 3 to $4-5$ and were as high as those detected in 'Success'. However, the $\mathrm{F} 3 \mathrm{H}$ protein was substantially less in both cultivars through all the developmental stages. Furthermore, at stage 6 , the four proteins were greatly reduced. In 'Spirit', greatly reduced protein levels were overall observed at stage 3 ; however, the levels increased over stages 4-1 to 6 except for DFR, where slightly reduced levels were observed. In 'Cheers', the CHS and DFR proteins were readily detected in all stages of spathe development, whereas the $\mathrm{F} 3 \mathrm{H}$ and ANS proteins were generally reduced except at stages 4-1 and 4-5, where high levels were noted. An antibody was also generated to target the F3' $\mathrm{H}$ protein in the spathe; however, it cross reacted with numerous proteins from the tissue and could not be used (data not shown).

\section{Discussion}

This study investigated for the first time the expression of important anthocyanin biosynthetic genes at the mRNA and protein levels and their association to anthocyanin abundance, phenotype, and genotype with the objective of understanding the molecular basis for differences in shade color in genetically characterized red and pink cultivars. Our findings showed that differences between red and pink cultivars, as well as differences between cultivars with various shades of pink, could be accounted for by the differences in the total anthocyanin content. Cultivars with pink spathes contained severely diminished anthocyanin levels in comparison with the red. In addition, among the pink-spathed cultivars, the anthocyanin abundance closely reflected the shade intensity indicated by $L^{*}$ (Figs. 2 and 3). Furthermore, the study showed that both temporal differences in the accumulation of anthocyanins in the spathe tissue as well as differences in the rate of anthocyanin accumulation over developmental time may have accounted for the final differences in spathe color.

It was found that shade differences among the pink cultivars can be phenotypically differentiated not only by $L^{*}$, which measured color intensity, but also by the $a^{*}$ color component. For instance, the $a^{*}$ dimension was high in the red and dark pink cultivars, Success and Sweety, respectively, but had lower values in the lighter pink cultivars. Additionally, there was a significant correlation $(P<0.05)$ between the anthocyanin levels and $a^{*}$ measurements (Fig. 4B). The differences in the $a^{*}$ parameter indicated that there were qualitative differences in the spathe colors, possibly due to variations in the combination of pigments present in the tissue. Studies have demonstrated that the differences among shades were determined not only by the concentration of anthocyanins (cyanidin and pelargonidin 3-rutinoside), but also by the ratio of cyanidin to pelargonidin (Iwata et al., 1985; Li et al., 2013). It was reported that a ratio $>1.0$ produced spathes with red and pink hues while $<1.0$ resulted in spathes with orange and coral hues. It may be possible that the reduced $F 3^{\prime} H$ transcripts in pinks resulted in carbon flux being partially channeled from dihydrokaempferol to leucopelargonidin and eventually the production of limited amounts of pelargonidin derivatives. Alternatively, the qualitative differences in color may be due to the effect of copigmentation. Flavones, which can exert a strong copigmentation effect with anthocyanins (Martens and Mithöfer, 2005), are produced in large amounts in the spathes of anthurium (Clark et al., 2012, 2014; Li et al., 2013). Avila-Rostant et al. (2011) noted that lighter shades of pink accumulated a greater abundance of total flavonoids compared with the darker pink and red spathes. It is possible that the accumulated flavonoids upstream of the bottlenecked $F 3^{\prime} H$ step of the pathway in pinks may be interacting with the anthocyanins.

Although there were significant differences in the transcript levels of all the genes between cultivars and stages, the transcript levels of the $C H S, F 3 H, D F R$, and $A N S$ genes did not correspond to shade color intensity $\left(L^{*}\right)$, quality $\left(a^{*}\right)$, or to the anthocyanin levels. Furthermore, their transcript levels declined over the development stages while anthocyanins and 

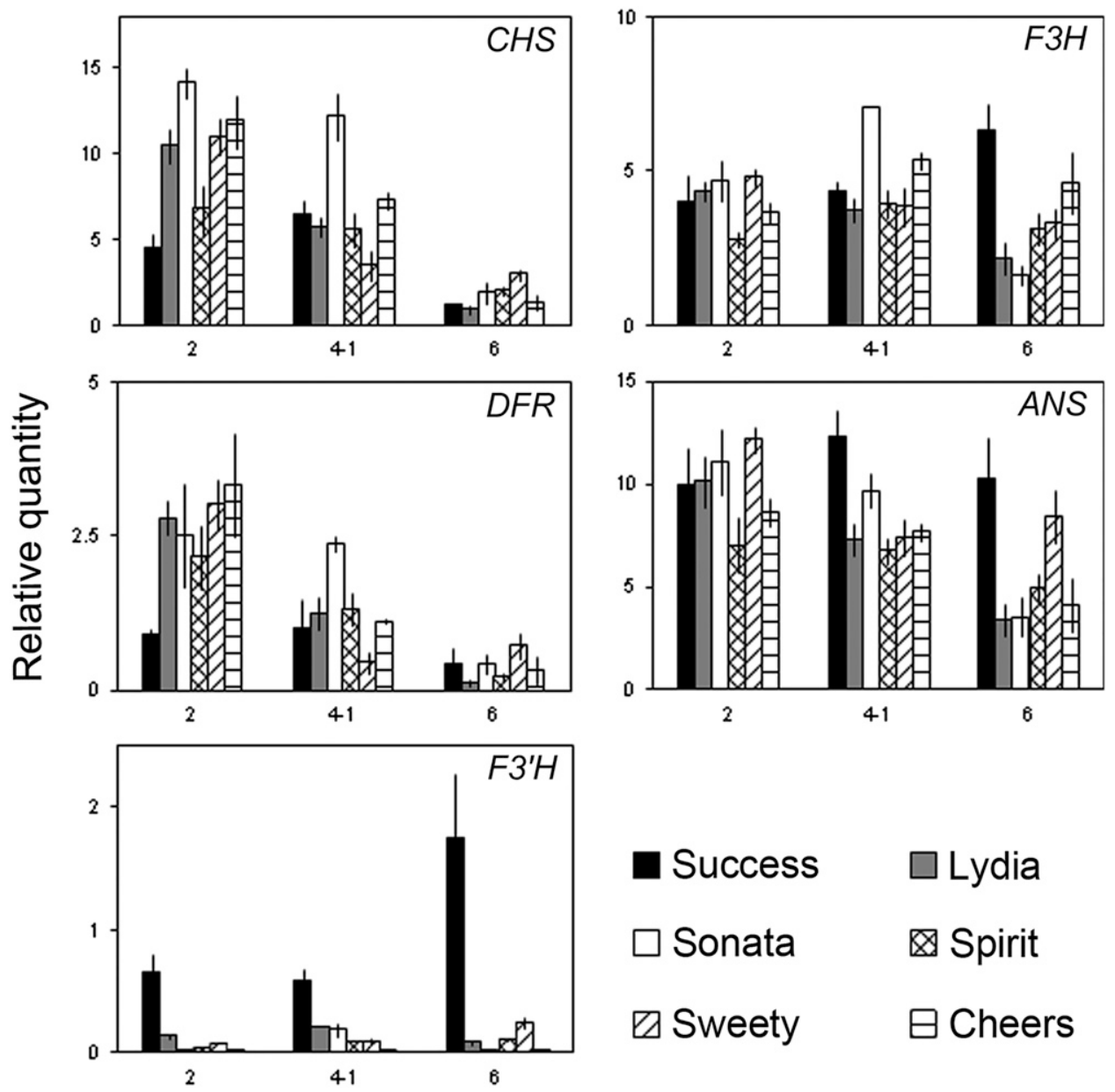

$\square$ Sonata



$\oslash$ Sweety $\boxminus$ Cheers

\section{Spathe development stage}

Fig. 5. Expression of chalcone synthase $(C H S)$, flavanone 3-hydroxylase $(F 3 H)$, dihydroflavonol 4-reductase $(D F R)$, anthocyanidin synthase $(A N S)$, and flavonoid $3^{\prime}$-hydroxylase $\left(F 3^{\prime} H\right)$ in the spathe tissue in six Anthurium andraeanum cultivars at spathe development stages 2, 4-1, and 6 determined using reverse transcription quantitative real-time polymerase chain reaction (PCR) analysis. Expression levels were normalized to the expression of cyclophilin and ubiquitin family protein. All data are presented as means of three biological replicates and error bars show SE.

color intensified. In contrast, the expression of $F 3^{\prime} H$ was significantly greater in the red-spathed cultivar compared with the pinks. Also, among the pinks, $F 3^{\prime} H$ mRNA was significantly higher in the darker pink than in the lighter pinks. The transcript level of $F 3^{\prime} H$ closely mirrored anthocyanin accumulation in these cultivars during spathe development suggesting that the expression of $F 3^{\prime} H$ may be involved in the differentiation between red and pinks as well as the differences in the intensity of the shade among pink spathes. In a recent study, it was proposed that in anthurium, $F 3^{\prime} H$ expression may be a key control point in the regulation of anthocyanin biosynthesis in red spathes (Gopaulchan et al., 2014) based on the correspondence between $F 3^{\prime} H$ expression and the intensity of red among red-spathed cultivars of anthurium. Previous studies have also noted a correspondence between the expression of $F 3^{\prime} H$ and the accumulation of anthocyanins. Brugliera et al. (1999) reported that the introduction of petunia's (Petunia hybrida) $F 3^{\prime} H$ gene into a cultivar that was deficient of $F 3^{\prime} H$ and flavonoid $3^{\prime}, 5^{\prime}$ hydroxylase $\left(F 3^{\prime} 5^{\prime} H\right)$ resulted in increased anthocyanin production, altering the flower color from pale pink to dark pink.
Nakatsuka et al. (2006) also observed an increase in the anthocyanin content and flower color intensity in tobacco (Nicotiana tabacum) with the expression of the gentian (Gentiana triflora) $G t F 3^{\prime} H$ gene. Similarly, Tanaka and Ohmiya (2008) noted the down regulation of $F 3^{\prime} H$ and $F 3^{\prime} 5^{\prime} H$ in torenia (Torenia hybrida), which typically accumulated delphinidin and cyanidin, produced a pale pink-colored transgenic containing mostly pelargonidin. Our results, along with these findings, suggest that the $F 3^{\prime} H$ expression may be involved in determining the shade color intensity in red and pink spathes. Further work is therefore required to evaluate this hypothesis.

Collette et al. (2004) reported that the expression of the anthocyanin genes peaked late during spathe development in pink spathes in comparison with the red spathes and hypothesized that temporal differences in expression of the genes were responsible for the color differences. The findings of this research also suggested that in addition to differences in overall transcript levels of $F 3^{\prime} H$, temporal differences in expression of the anthocyanin genes, in particular $F 3^{\prime} H$, may have also contributed to the differences in anthocyanin abundance and color intensity among the pink-spathed cultivars (Figs. 5 and 6). Temporal regulation of the anthocyanin biosynthetic genes during organ development and its influence on color diversity and intensity, have also been noted in other species. For instance in orchid (Cymbidium hybrida), Wang et al. (2014) reported that the temporal and spatial expression of $D F R$ and $A N S$ were responsible for the different flower color groups and the diversity of colors and shades within a group. In Clarkia gracilis, the difference in pigment types between the petals and petal spots, as well as spatial restriction of the spots, has been proposed to be due to the different temporal expression patterns of $F 3^{\prime} H 1-A$ and $D F R 2$ genes and the $F 3^{\prime} 5^{\prime} H 1$ and $D F R 1$ genes (Martins et al., 2013). In some species, such as maize (Zea mays), the genes encoding the enzymes of the anthocyanin biosynthetic pathway are expressed simultaneously during development and are activated by a common regulatory mechanism consisting of R2R3 MYB and basic helix-loophelix type transcription factors (Petroni et al., 2014; Petroni and Tonelli, 2011). In other species, such as snapdragon (Antirrhinum majus), the genes are separated into two regulatory units: the early biosynthetic genes (EBGs) and the late biosynthetic genes (LBGs) (Jackson et al., 1992; Martin et al., 1991). The EBGs are expressed during early stages of development, 

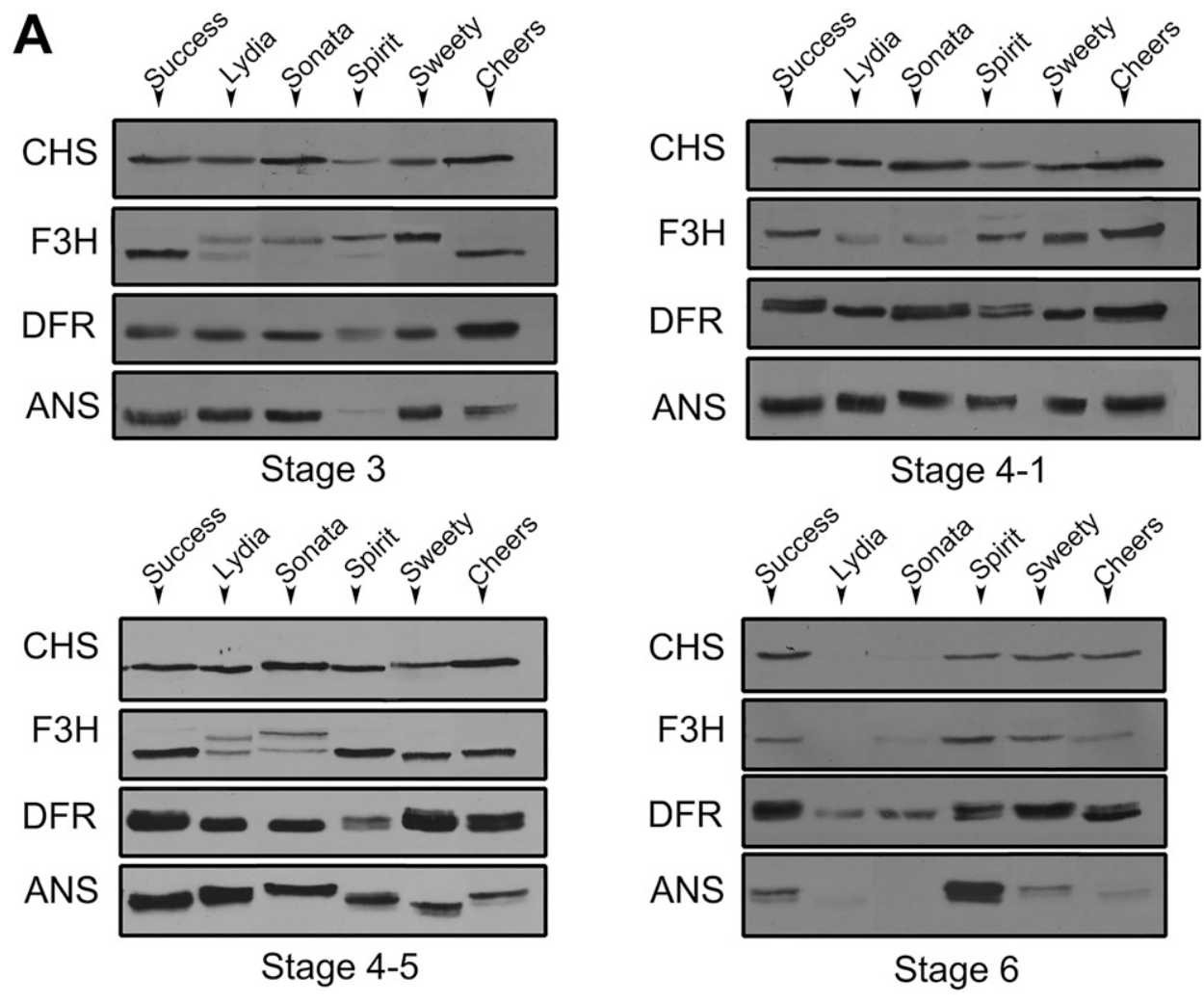

The mRNA levels of $F 3 H$ in all five pink-spathed cultivars were similar to Success, a red-spathed cultivar. Nevertheless, in the pink cultivars, Lydia and Sonata, the $\mathrm{F} 3 \mathrm{H}$ protein abundance was severely diminished throughout spathe development and in the pink cultivar, Cheers, there was a reduction in both $\mathrm{F} 3 \mathrm{H}$ and ANS protein. $\mathrm{F} 3 \mathrm{H}$ is an essential enzyme in the anthocyanin biochemical pathway that catalyzes the conversion of naringenin to dihydrokaempferol, an important precursor for anthocyanin formation. It is likely the reduced $\mathrm{F} 3 \mathrm{H}$ protein could have acted as a rate-limiting step and restricted the movement of carbon flux through the pathway hindering anthocyanin production in these cultivars. Zabala and Vodkin (2005) noted that in soybean (Glycine max) a transposon insertion in the $\mathrm{F} 3 \mathrm{Hl}$ gene resulted in reduced $\mathrm{F} 3 \mathrm{H}$ levels and the occurrence of pinkcolored flowers instead of purple. However, sequence analysis of the anthurium $\mathrm{F} 3 \mathrm{H}$ gene did not reveal such insertions or any mutations that could account for the reduced protein level (data not presented). Studies have shown $F 3 H$ belongs to a multigene family and that different isoforms might be expressed to produce the overall enzyme activity (Azuma et al., 2012; Shen et al., 2006). In anthurium, two other putative $F 3 H$ isoforms with different DNA sequences have been discovered; however, their transcript levels were negligible in comparison with the isoform studied in this research (unpublished work). This suggests that the assayed $F 3 H$ is the active isoform in the anthocyanin biosynthetic pathway. It is possible that the low-protein abundance of $\mathrm{F} 3 \mathrm{H}$ in some pink cultivars may be a result of the gene being regulated at the post-transcriptional level, as has been reported for other genes in the flavonoid biosynthetic path-

whereas the LBGs are expressed at a later stage. However, in anthurium, although the anthocyanin genes (CHS, DFR, F3H, and $A N S$ ) are coexpressed, they are controlled by independent regulatory mechanisms (Avila-Rostant et al., 2011; Gopaulchan et al., 2014). It is plausible that a common stimulus or combination of stimuli induce the expression of the regulatory genes, which act in concert to coordinate the expression of the anthocyanin genes during spathe development. way. For instance, in maize, the In locus tightly regulates the expression of $C H S$ at the post-transcriptional level (Franken et al., 1991). Burbulis and Winkel-Shirley (1999) proposed that $\mathrm{CHI}$ in arabidopsis (Arabidopsis thaliana) was subjected to post-translation processing. Pairoba and Walbot (2003) found that the $B z 2$ gene in maize, which encodes glutathione S-transferase, was also strictly regulated at the post-transcriptional level. 
In the mature spathes of 'Lydia' and 'Sonata', a reduction of anthocyanin abundance was noted compared with the earlier stages. This reduction coincided with diminished expression of the anthocyanin biosynthetic genes at both the transcript and protein levels, indicating that the biosynthesis of anthocyanins was suppressed. Additionally, pigment dilution during spathe growth and expansion, as well as anthocyanin degradation, may have also led to the reduced concentrations (Oren-Shamir, 2009). On the contrary, 'Sweety' and 'Spirit' accumulated anthocyanins late into spathe development, albeit at different rates, which correlated with late expression of the anthocyanin biosynthetic genes. It is possible that the greater increase in anthocyanin abundance and darker shade of pink noted in 'Sweety' in comparison with 'Spirit' in the mature spathe was likely due to the higher $F 3^{\prime} H$ transcripts noted at the later stages.

Kamemoto et al. (1988) theorized that the dosages of the $M$ and $O$ loci were responsible for the range of shade colors and that pink cultivars were heterozygous for the $M$ and $O$. It was later proposed by Elibox and Umaharan (2008) that the $M$ locus encoded the $F 3^{\prime} H$ gene, which controls the shift from dihydrokaempferol, the precursor for pelargonidin derivatives, to dihydroquercetin, the precursor for cyanidin derivatives. The study further suggested that the dosage of the $R$ gene corresponded to the differences between reds and pinks. However, in this study, among the cultivars evaluated, we did not observe a dosage effect of the $M$ locus $(M M$ or $M m)$ on the final phenotype of the pink as observed by Kamemoto et al. (1988). For instance, the anthocyanin content and the corresponding $F 3^{\prime} H$ transcript level in 'Lydia' and 'Sonata' that were $M M$ were significantly less than 'Success' that was $\mathrm{Mm}$. Similarly, 'Sweety', which was $M m$, exhibited greater $F 3$ ' $H$ transcript and anthocyanin abundance than 'Lydia' and 'Sonata'. Also noticeable was the distinct expression profile of $F 3^{\prime} H$ in comparison with the $C H S, F 3 H, D F R$, and $A N S$ genes, indicating that $F 3^{\prime} H$ was controlled separately from the other biosynthetic genes by another regulatory mechanism. These results suggest that while the $M$ locus may encode the $F 3^{\prime} H$ gene, another gene or genes (unaccounted for by the genetic model) may encode a transcription factor(s) that regulates the expression of $F 3^{\prime} H$. These transcription factor(s) may therefore control the intensity of shade color in anthurium by affecting the temporal expression and the level of expression of the $F 3^{\prime} H$ gene, as was evident in this study.

There was evidence of other mechanisms, other than that mediated through the $F 3^{\prime} H$ gene, which may have contributed toward the differences between red and pink-spathed cultivars. The reduced expression of $F 3 H$ in 'Lydia' and 'Sonata', F3H and $A N S$ in 'Cheers' particularly during the early development stages, and $D F R$ in 'Spirit', also appeared to be involved in determining the final color. Further study may provide evidence for the dosage effects seen for the $O$ and $R$ genes (Elibox and Umaharan, 2008; Kamemoto et al., 1988). This multitude of mechanisms for color variation may reflect the complex nature of the ornamental, which has been developed through interspecific hybridization between species of the sections Calomystrium and Porphyrochitonium.

In conclusion, anthurium is a monocot with a highly pigmented spathe, which is a modified leaf with an extended developmental period. The study shows that differences between red- and pink-colored spathes as well as differences among the shade intensities of pink spathes could be explained by differences in their relative anthocyanin abundances. Furthermore, differences in anthocyanin levels among pinks were correlated to differences in the level of $F 3^{\prime} H$ expression. $F 3^{\prime} H$ gene expression has similarly been shown to be correlated to shade differences among red cultivars in anthurium (Gopaulchan et al., 2014). There is also evidence from the study that the greatly diminished levels of anthocyanins in pink cultivars could be due to additional regulatory mechanisms. The study showed that in addition to reduced transcript levels of $F 3^{\prime} H$, there were diminished levels of F3H, ANS, or DFR proteins in pink cultivars, which could account for the reduced carbon flux through the flavonoid pathway. While correlation between gene expression and anthocyanin contents provide preliminary evidence of the role of these genes in determining spathe color intensity, further validatory work is required to clearly elucidate the roles of these genes on shade intensity differences among pinks.

\section{Literature Cited}

Avila-Rostant, O., A.M. Lennon, V.E. Collette, and P. Umaharan. 2011. Determination of expression patterns of flavonoid biosynthetic genes in cultivars of Anthurium andraeanum towards understanding regulatory control of spathe colour. Trop. Agr. (Trinidad) 88:10-17. Azuma, A., H. Yakushiji, Y. Koshita, and S. Kobayashi. 2012. Flavonoid biosynthesis-related genes in grape skin are differentially regulated by temperature and light conditions. Planta 236:10671080 .

Bradford, M.M. 1976. A rapid and sensitive method for the quantitation of microgram quantities of protein utilizing the principle of protein-dye binding. Anal. Biochem. 72:248-254.

Brugliera, F., G. Barri-Rewell, T.A. Holton, and J.G. Mason. 1999. Isolation and characterization of a flavonoid 3 '-hydroxylase cDNA clone corresponding to the Ht1 locus of Petunia hybrida. Plant J. 19:441-451.

Burbulis, I.E. and B. Winkel-Shirley. 1999. Interactions among enzymes of the Arabidopsis flavonoid biosynthetic pathway. Proc. Natl. Acad. Sci. USA 96:12929-12934.

Clark, B.R., B.J. Bliss, J.Y. Suzuki, and R.P. Borris. 2014. Chemotaxonomy of Hawaiian Anthurium cultivars based on multivariate analysis of phenolic metabolites. J. Agr. Food Chem. 62:11323-11334.

Clark, B.R., J.Y. Swzuki, B.J. Bliss, and R.P. Borris. 2012. Flavone $C$ glycosides from Anthurium andraeanum. Natl. Prod. Commun. 7:747-748.

Collette, V.E., P.E. Jameson, K.E. Schwinn, P. Umaharan, and K.M. Davies. 2004. Temporal and spatial expression of flavonoid biosynthetic genes in flowers of Anthurium andraeanum. Physiol. Plant. 122:297-304.

Elibox, W. and P. Umaharan. 2008. Inheritance of major spathe colors in Anthurium andraeanum Hort. is determined by three major genes. HortScience 43:787-791.

Franken, P., U. Niesbach-Klösgen, U. Weydemann, L. MaréchalDrouard, H. Saedler, and U. Wienand. 1991. The duplicated chalcone synthase genes $C 2$ and $W h p$ (white pollen) of Zea mays are independently regulated; Evidence for translational control of $W h p$ expression by the anthocyanin intensifying gene in. EMBO J. 10:2605-2612.

Gopaulchan, D., A.M. Lennon, and P. Umaharan. 2013. Identification of reference genes for expression studies using quantitative RT-PCR in spathe tissue of Anthurium andraeanum (Hort.). Sci. Hort. 153:1-7. Gopaulchan, D., P. Umaharan, and A.M. Lennon. 2014. A molecular assessment of the genetic model of spathe color inheritance in Anthurium andraeanum (Hort.). Planta 239:695-705.

Harlow, E. and D. Lane. 1988. Antibodies: A laboratory manual. Cold Spring Harbor Lab., Cold Spring Harbor, NY.

Iwata, R.Y., C. S. Tang, and H. Kamemoto. 1979. Anthocyanins of Anthurium andreanum Lind. J. Amer. Soc. Hort. Sci. 104:464-466. 
Iwata, R.Y., C. S. Tang, and H. Kamemoto. 1985. Concentration of anthocyanins affecting spathe color in anthuriums. J. Amer. Soc. Hort. Sci. 110:383-385.

Jackson, D., K. Roberts, and C. Martin. 1992. Temporal and spatial control of expression of anthocyanin biosynthetic genes in developing flowers of Antirrhinum majus. Plant J. 2:425-434.

Kamemoto, H., R.Y. Iwata, and M. Marutani. 1988. Genetics of the major spathe colors in anthuriums. HITAHR College Trop. Agr. Human Resources Res. Ser. 056:8-88.

Laemmli, U.K. 1970. Cleavage of structural proteins during the assembly of the head of bacteriophage T4. Nature 227:680-685.

Lee, J., R.W. Durst, and R.E. Wrolstad. 2005. Determination of total monomeric anthocyanin pigment content of fruit juices, beverages, natural colorants, and wines by the $\mathrm{pH}$ differential method: Collaborative study. J. AOAC Intl. 88:1269-1278.

Li, C., G. Yang, S. Huang, D. Lü, C. Wang, J. Chen, and J. Yin. 2013. Characterisation of flavonoids in anthurium spathes and their contribution to spathe colouration. J. Hort. Sci. Biotechnol. 88:208-215.

Lukačin, R., I. Gröning, E. Schiltz, L. Britsch, and U. Matern. 2000. Purification of recombinant flavanone $3 \beta$-hydroxylase from Petunia hybrida and assignment of the primary site of proteolytic degradation. Arch. Biochem. Biophys. 375:364-370.

Martens, S. and A. Mithöfer. 2005. Flavones and flavone synthases. Phytochemistry 66:2399-2407.

Martin, C., A. Prescott, S. Mackay, J. Bartlett, and E. Vrijlandt. 1991. Control of anthocyanin biosynthesis in flowers of Antirrhinum majus. Plant J. 1:37-49.

Martins, T.R., J.J. Berg, S. Blinka, M.D. Rausher, and D.A. Baum. 2013. Precise spatio-temporal regulation of the anthocyanin biosynthetic pathway leads to petal spot formation in Clarkia gracilis (Onagraceae). New Phytol. 197:958-969.

Nakatsuka, T., M. Nishihara, K. Mishiba, and S. Yamamura. 2006. Heterologous expression of two gentian cytochrome P450 genes can modulate the intensity of flower pigmentation in transgenic tobacco plants. Mol. Breed. 17:91-99.
Oren-Shamir, M. 2009. Does anthocyanin degradation play a significant role in determining pigment concentration in plants? Plant Sci. 177:310-316.

Pairoba, C.F. and V. Walbot. 2003. Post-transcriptional regulation of expression of the Bronze2 gene of Zea mays L. Plant Mol. Biol. 53:75-86.

Pelletier, M.K., I.E. Burbulis, and B. Winkel-Shirley. 1999. Disruption of specific flavonoid genes enhances the accumulation of flavonoid enzymes and end-products in Arabidopsis seedlings. Plant Mol. Biol. 40:45-54.

Petroni, K., R. Pilu, and C. Tonelli. 2014. Anthocyanins in corn: A wealth of genes for human health. Planta 240:901-911.

Petroni, K. and C. Tonelli. 2011. Recent advances on the regulation of anthocyanin synthesis in reproductive organs. Plant Sci. 181:219229.

Rieu, I. and S.J. Powers. 2009. Real-time quantitative RT-PCR: Design, calculations, and statistics. Plant Cell 21:1031-1033.

Schijlen, E.G.W.M., C.H.R.d. Vos, A.J.v. Tunen, and A.G. Bovy. 2004. Modification of flavonoid biosynthesis in crop plants. Phytochemistry 65:2631-2648.

Schneider, C.A., W.S. Rasband, and K.W. Eliceiri. 2012. NIH Image to ImageJ: 25 years of image analysis. Natl. Methods 9:671-675.

Shen, G., Y. Pang, W. Wu, Z. Deng, L. Zhao, Y. Cao, X. Sun, and K. Tang. 2006. Cloning and characterization of a flavanone 3-hydroxylase gene from Ginkgo biloba. Biosci. Rpt. 26:19-29.

Tanaka, Y. and A. Ohmiya. 2008. Seeing is believing: Engineering anthocyanin and carotenoid biosynthetic pathways. Curr. Opin. Biotechnol. 19:190-197.

Wang, L., N.W. Albert, H. Zhang, S. Arathoon, M.R. Boase, H. Ngo, K.E. Schwinn, K.M. Davies, and D.H. Lewis. 2014. Temporal and spatial regulation of anthocyanin biosynthesis provide diverse flower colour intensities and patterning in Cymbidium orchid. Planta 240:983-1002.

Zabala, G. and L.O. Vodkin. 2005. The $w p$ mutation of Glycine max carries a gene-fragment-rich transposon of the CACTA superfamily. Plant Cell 17:2619-2632. 